\title{
Drift velocity in n-pentane mixtures and its influence on timing properties of Thin Gap Chambers
}

\author{
D. Lazic,${ }^{1}$ N. Lupu, A. Mincer ${ }^{2}$, Y. Rozen, S. Tare ${ }^{--}$ \\ Physics Department, Technion - Israel Institute of Technology, \\ Technion City, 32000 Haifa, Israel.
}

\author{
A. Breskin, R. Chechik, D. Lellouch, G. Mala- ud ${ }^{3}$, \\ G. Mikenberg, K. Nagai, A. Pansky ${ }^{4}$, M. Shoa \\ Department of Particle Physics, The Weizmann Institute of Science, \\ 76100 Rehovot, Israel.
}

\begin{abstract}
We report on measurements of the electron drift velocity and longitudinal diffusion in n-pentane gas mixtures and on the effect of the drift velocity on the timing properties of Thin-Gap-Chambers. Gas mixtures of n-pentane- $\mathrm{CO}_{2}$, n-pentane$\mathrm{CO}_{2}-\mathrm{CH}_{4}$, and n-pentane- $-\mathrm{CO}_{2}-\mathrm{CF}_{4}$ were investigated.

An increase of the drift velocity has been observed with mixtures containing $\mathrm{CF}_{4}$, while still maintaining the very high, saturated gain, typical for these chambers. The overall improvement of TGC timing properties is such that $99 \%$ detection efficiency can be reached within a 20 ns gate.

A simulation of the chamber timing properties using the measured drift velocities, reproduces well the measured data and can be used to predict chamber performance as a function of geometry and gas mixture.
\end{abstract}

\footnotetext{
${ }^{1}$ Corresponding author. E-mail addres: Dragoslav.La-ic@cern.ch

${ }^{2}$ Permanent address, New York University, New York, NY, USA

${ }^{3}$ Present address, Elscint, Haifa, Israel

${ }^{4}$ Present address, Elgems, Haifa, Israel
} 


\section{Introduction}

The detection of a charged lepton $[1,2]$ is the primary trigger for many LHC processes. The high luminosity imposes rather stringent requirements on the trigger detector performance. The muon trigger system has to provide a fast digital signal within a $25 \mathrm{~ns}$ gate defined by the interval between beam crossings.

Thin gap chambers (TGC's) [3,4] operating with n-pentane- $\mathrm{CO}_{2}$ mixture, have given very good results in long-term operation $[3,4]$ in the OPAL experiment of the CERN Large Electron Positron Collider (LEP) [5,6] and were subsequently chosen for muon trigger chambers in the endcap region of the LHC experiment ATLAS.

The TGCs are well suited for the LHC as they operate in a saturated proportional mode, giving fast signals with typical rise times below $5 \mathrm{~ns}$. A saturated mode of operation leads to strong signals with reduced Landau tails and high signal-to-noise ratio. Therefore, with simple electronics, the TGC can easily satisfy the muon trigger requirements in the endcap region. The saturated proportional mode is achieved under a strong electric field in a $55 \% \mathrm{CO}_{2} / 45 \%$ n-pentane gas mixture. This mixture is easily obtained by bubbling the $\mathrm{CO}_{2}$ at atmospheric pressure through liquid n-pentane at $17^{\circ} \mathrm{C}$.

To ensure compliance of the TGC with the fast timing requirement at the LHC, a computer simulation study of the detection processes was undertaken. One of the basic input variables necessary in such simulations is the drift velocity of electrons in the working gas. The value of this velocity was unavailable in the literature and its measurement, along with the associated diffusion coefficient, are reported here. In addition, the characteristics of other gas mixtures that can potentially improve the chamber operation were also measured. The effects of these gas mixtures on TGC operation were tested and compared to simulation results.

Although the $55 \% \mathrm{CO}_{2} / 45 \%$ n-pentane mixture has many advantages, it results in relatively slow electron drift. The actual fast time resolution is achieved in the TGC chambers by their small anode to cathode distance and large electric fields. The measured drift velocity and diffusion coefficient of the two component gas mixture were compared to the characteristics of similar mixtures containing additional gas species. This was done in order to check if such additions can potentially improve the chamber operation by increasing drift velocity.

In the following sections we will describe the structure of the chambers (Section 2), the measurements of the gas mixtures characteristics (Section 3), the timing properties of the chambers (Section 4) and the simulation of TGC timing 


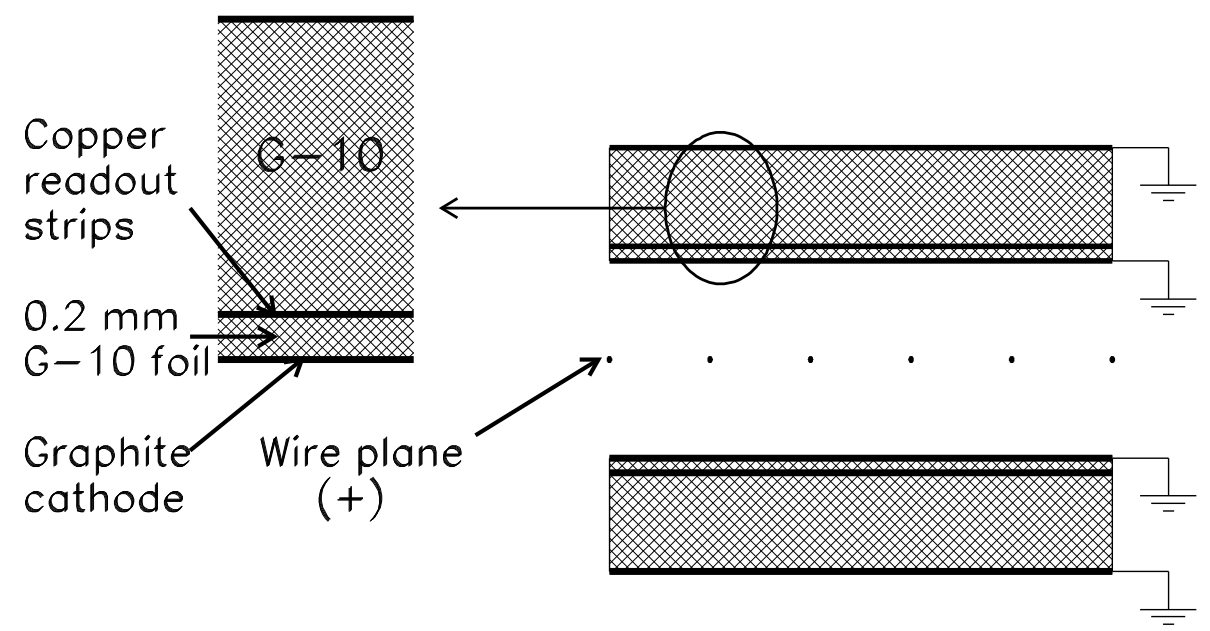

Fig. 1. Thin gap chamber general layout. See text for dimensions of the prototypes used in different measurements.

properties (section 5).

\section{TGC Structure and timing properties}

Thin gap chambers have a structure similar to that of Multiwire Proportional Chambers, except that the anode-to-anode distance is larger than that of the anode-to-cathode (see Figure 1 and Refs. [3-7]). The ATLAS prototypes have an anode plane made of $50 \mu \mathrm{m}$ in diameter gold-plated tungsten wires with $1.8 \mathrm{~mm}$ pitch. The wire plane is sandwiched between graphite cathodes $1.40 \mathrm{~mm}$ from the wires. $9 \mathrm{~mm}$ wide copper strips with $10 \mathrm{~mm}$ pitch, separated by $200 \mu \mathrm{m}$ thick G-10 foil from the cathode, provide a capacitively coupled readout. The mechanical support is provided by $1.6 \mathrm{~mm}$ thick G-10 plates that carry the strips. Wires are grouped together.

The signal arrival time in the TGC is dominated by the drift of electrons. It has been observed[7] that the usual tail in the signal arrival time distribution in TGCs originates from those particles that pass through the region close to the boundary between two wires, where the electric field, and consequently the electron drift velocity, are very low. This motivated the decrease in size of the elementary cell in the present prototype, compared to that used in the OPAL experiment[5,6]. The reduction of the relative size of the "slow" drift region leads indeed to somewhat shorter tails of the timing distributions as shown in figure 9-3 and 9-4 of [8]. 
The timing distributions and consequently the trigger performance, therefore, depend strongly on the electron drift velocity. Even a small increase of the electron drift velocity can lead to improved timing, especially for the regions of low electric field. Therefore, the possibility of improving the chamber performance through use of a different working gas has also been investigated.

In order to retain a high gain and stability, $\mathrm{CO}_{2}$ was kept as the working gas and n-pentane as a quencher. A third gas, either $\mathrm{CF}_{4}$ or $\mathrm{CH}_{4}$, with very high drift velocity at low values of electric field, was added to the mixture. The fraction of the additive gas had to be kept low because of the flammability of $\mathrm{CH}_{4}$.

\section{Measurement of the drift velocity}

The electron transport properties were measured with a Low-Pressure Multistep Gas Chamber (LPMSC), preceded by a long drift volume $[9,10]$. These devices have high sensitivity to single electrons and operate under low potentials at pressure of a few tens of Torr. The obtained values of drift velocity and longitudinal diffusion were scaled to the atmospheric pressure.

The layout of the LPMSC used in our measurements is shown in Figure 2. It consists of two main sections: drift and amplification volumes. The drift volume is $9.74 \mathrm{~cm}$ long. An electric field is applied across this volume by setting the first grid at a high negative potential and keeping the second grid (that coincides with one of the grids of the first amplification section) at ground potential. Field-shaping electrodes were built in the walls of the drift volume, ensuring the field homogeneity. A collimated pulsed ultraviolet (UV) lamp was used to illuminate the detector volume through a quartz window, inducing single photoelectrons emitted from various mesh electrodes. The obtained charges from meshes 1 and 2 (see figure 2) followed a full two-stage multiplication process, obtained by setting appropriate potential differences between meshes 2 to 3 and 4 to 5 , and were detected. The amplification of the charges was performed in two steps, separated by a transfer region to reduce secondary effects. The total amplification was of the order of $10^{6}$, sufficient for efficiently detecting individual electrons. Through electric pickup the UV lamp pulses triggered a digitizing oscilloscope, which recorded the obtained pulse trains and transferred the data to a PC. The pulses were analyzed on-line and single electron peaks were recognized by correlating them with a typical pulse shape[10,11].

As mentioned above, the detected pulse trains consisted of signals from charges produced in different sections of the conversion volume. The first electrons to arrive in the amplification region are those produced by photoeffect in 


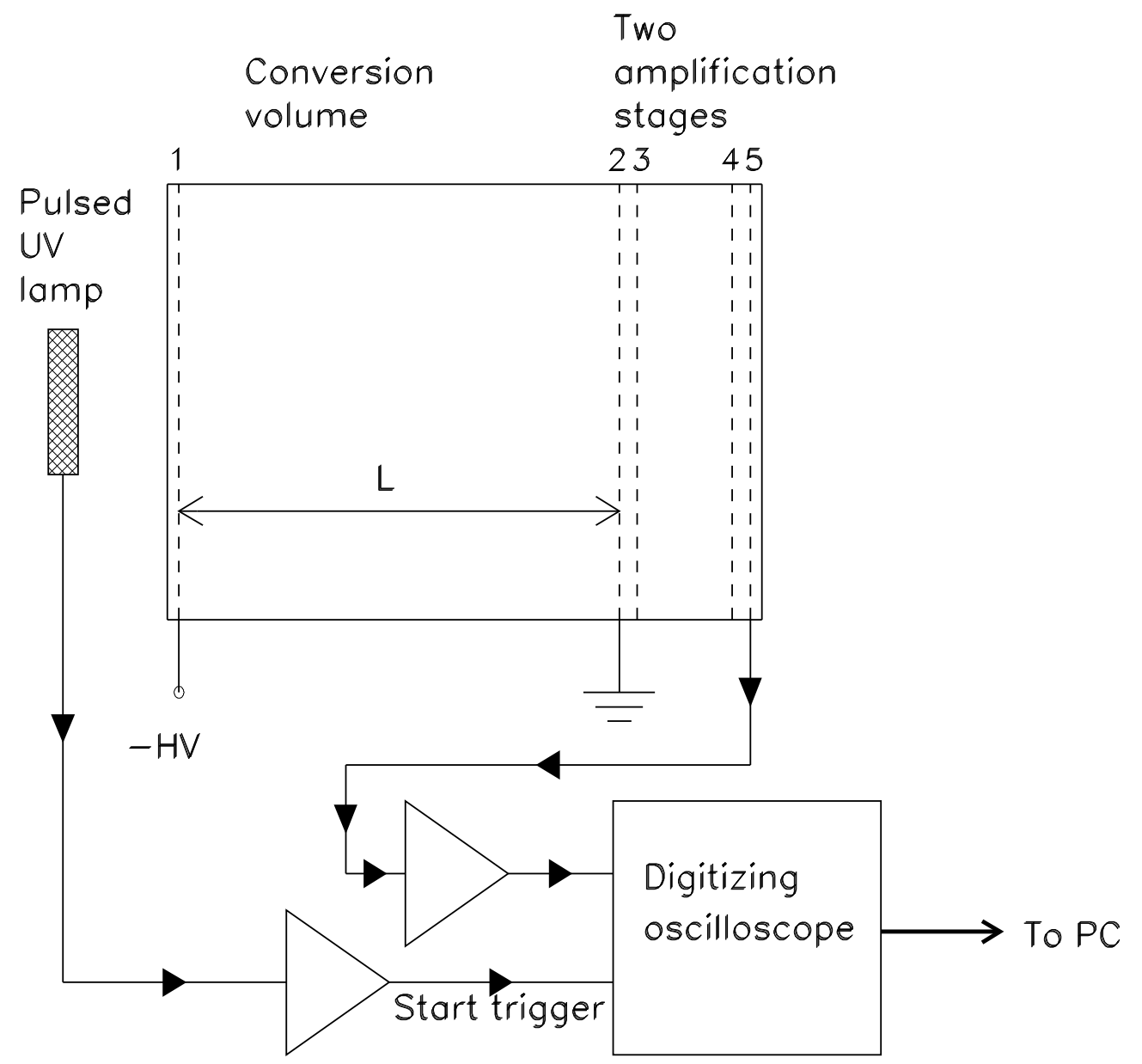

Fig. 2. The layout of the Low Pressure Multistep Gas Chamber used for electron drift velocity and diffusion measurements.

the first amplification stage cathode grid (2). The last electrons to arrive are those produced by photoeffect on the high-voltage grid (1). No signals appear from photoeffects on the other mesh electrodes because these electrons do not undergo the preamplification process. By dividing the length of the drift volume, $L$, by the time difference, $\Delta t$, between the pulses from electrodes 1 and 2 , we get the value of the drift velocity for the given value of electric field: $V_{\text {drift }}=L / \Delta t$. The longitudinal diffusion, $\sigma_{l}$, scaled to atmospheric pressure and to $1 \mathrm{~cm}$ drift path, is found from the r.m.s of the time difference between pulses, $\sigma(\Delta t)$, using $\sigma_{l}=\sigma(\Delta t) \cdot V_{\mathrm{drift}} \cdot \sqrt{\frac{p}{760 \cdot L}}$, where $p$ is the pressure in Torr.

The measured values of drift velocity and longitudinal diffusion are summarized in Figures 3 a) and b) respectively. Figure 3a) shows that with the addition of $\mathrm{CH}_{4}$ the drift velocity increases slightly in the low field region only. However, the introduction of $\mathrm{CF}_{4}$ has a rather dramatic effect: an increase of 


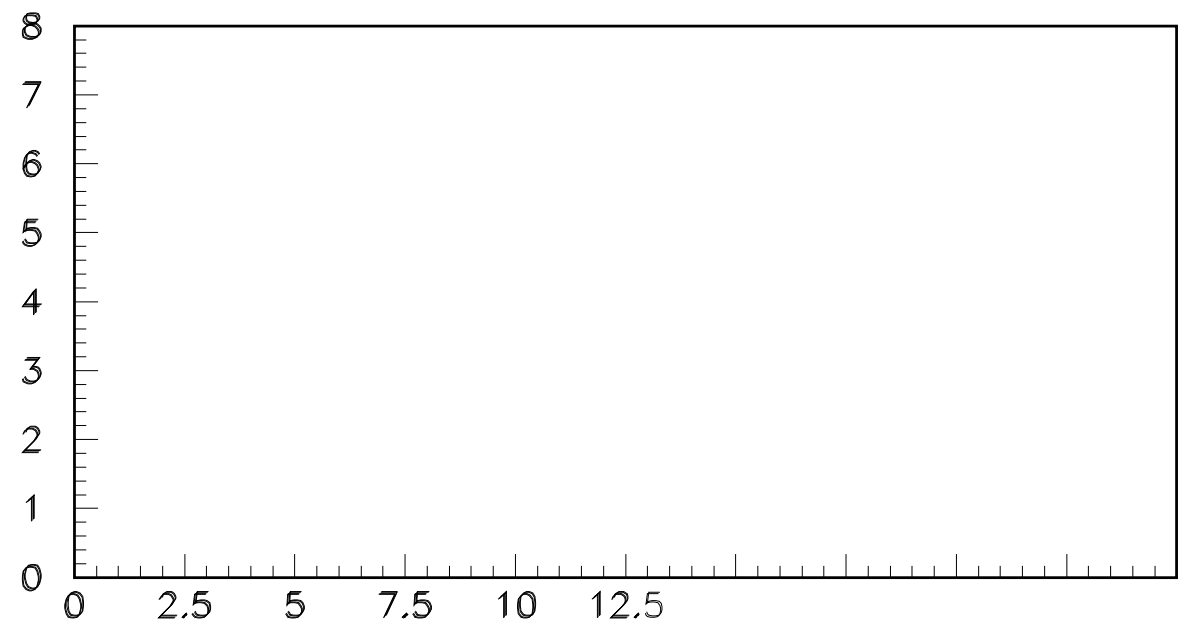




\section{TGC time response measurements with different gas mixtures}

We have measured the time response of a TGC operating with some of the gas mixtures investigated above. The time distribution measurements were performed by irradiating a TGC prototype with electrons from a collimated ${ }^{106} \mathrm{Ru}$ radioactive source. The prototype used was a small chamber $\left(10 \times 10 \mathrm{~cm}^{2}\right)$ with a structure as described in section 2 . All the wires of this prototype were electrically interconnected. The time response was measured versus a plastic scintillator placed behind the TGC. Its signal was used to trigger the VME-based data acquisition system. The signals from the chamber were amplified by a fast amplifier (Ortec FTA 820) and discriminated (Le Croy 4608B). Their arrival time was digitized in a Le Croy 2228 A TDC. An on-line data-acquisition system carried out the statistical analysis of the obtained distributions.

The chambers were tested with two values of high-voltage $(3 \mathrm{kV}$ and $3.2 \mathrm{kV})$ and several $\mathrm{CF}_{4}$-based gas mixtures. These voltages were low enough to avoid streamer formarion and spontaneous discharging of the chamber, but were still high enough to reach the saturated proportional mode of operation. The n-pentane content was maintained at $45 \%$ in all the mixtures, and only the fraction of $\mathrm{CF}_{4}$ and, consequently, that of $\mathrm{CO}_{2}$ were changed.

Figure 4 shows the arrival time distribution for TGC signals running at $3.0 \mathrm{kV}$ with three mixtures containing different $\mathrm{CF}_{4}$ fractions. It can be observed that the distribution narrows and the long time tail is reduced when $\mathrm{CF}_{4}$ is added to the mixture. The distributions do not change significantly for mixtures with over $20 \% \mathrm{CF}_{4}$. The corresponding TGC efficiency within 20 and $25 \mathrm{~ns}$ windows are summarized in Figure 5. The efficiency of $99 \%$ within a 25 ns gate is obtained with the original gas mixture. The addition of $\mathrm{CF}_{4}$ improves the efficiency, reaching over $99 \%$ within a 20 ns gate for mixtures with $20 \% \mathrm{CF}_{4}$ or more. The increase of the $\mathrm{CF}_{4}$ content led to an increase of the pulse height. These results are encouraging, showing that introducing the correct amount of $\mathrm{CF} 4$, one can speed up the chamber response without compromising the saturated regime of operation or the overall detector efficiency.

\section{Simulation of TGC timing properties}

The measured values of the drift velocity were used to perform Monte Carlo simulations of the chamber time response. The electron drift time from each point in the detector to the wire was calculated with the GARFIELD[12] program package (see Figure 6), using the chamber geometry, applied high voltage, and the measured values of the drift velocity as input parameters. The obtained drift times were stored in two-dimensional look-up tables. Figure 

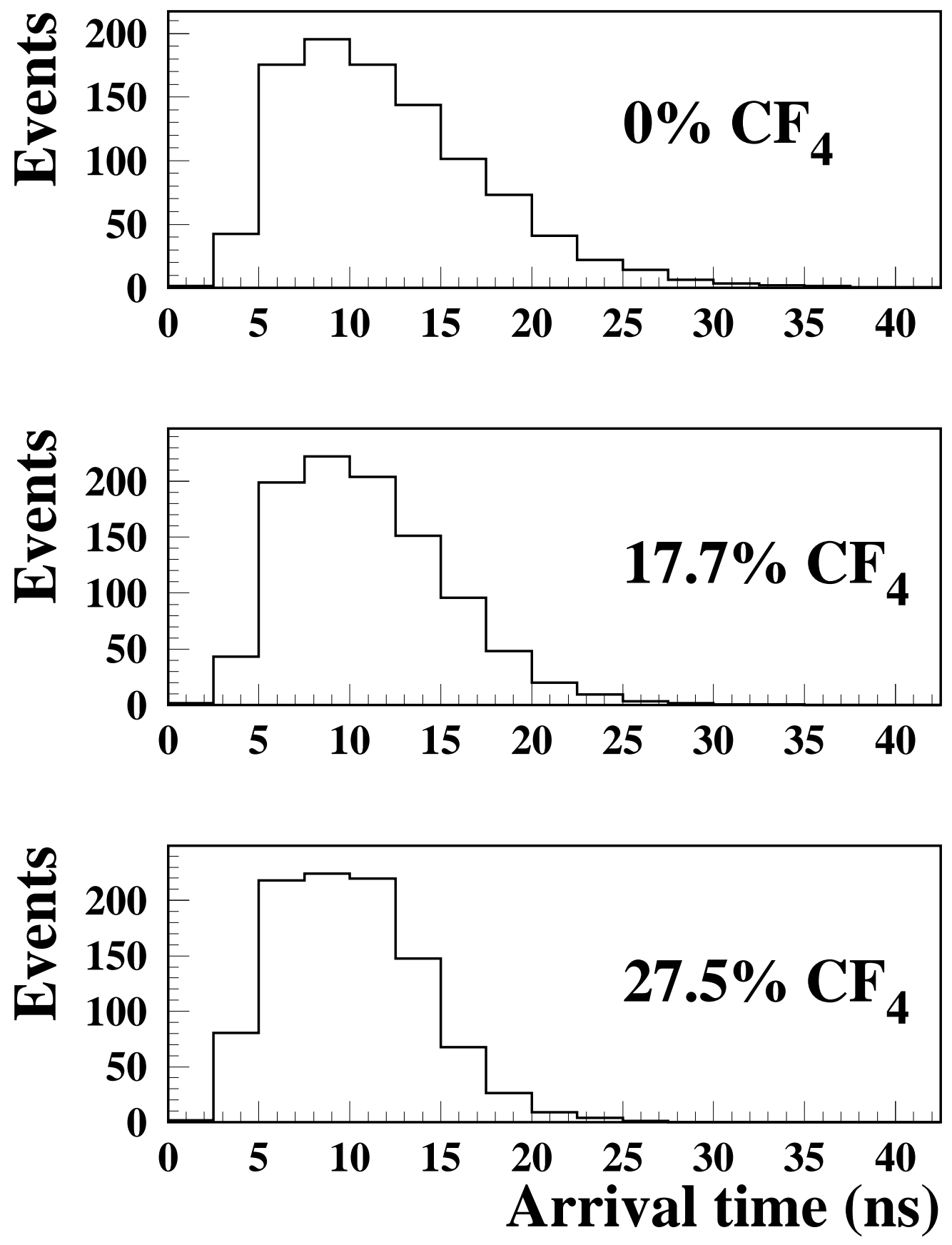

Fig. 4. Distribution of arrival times of the signals from TGC as a function of the $\mathrm{CF}_{4}$ concentration. The n-pentane contegt has been kept at $45 \%$. 


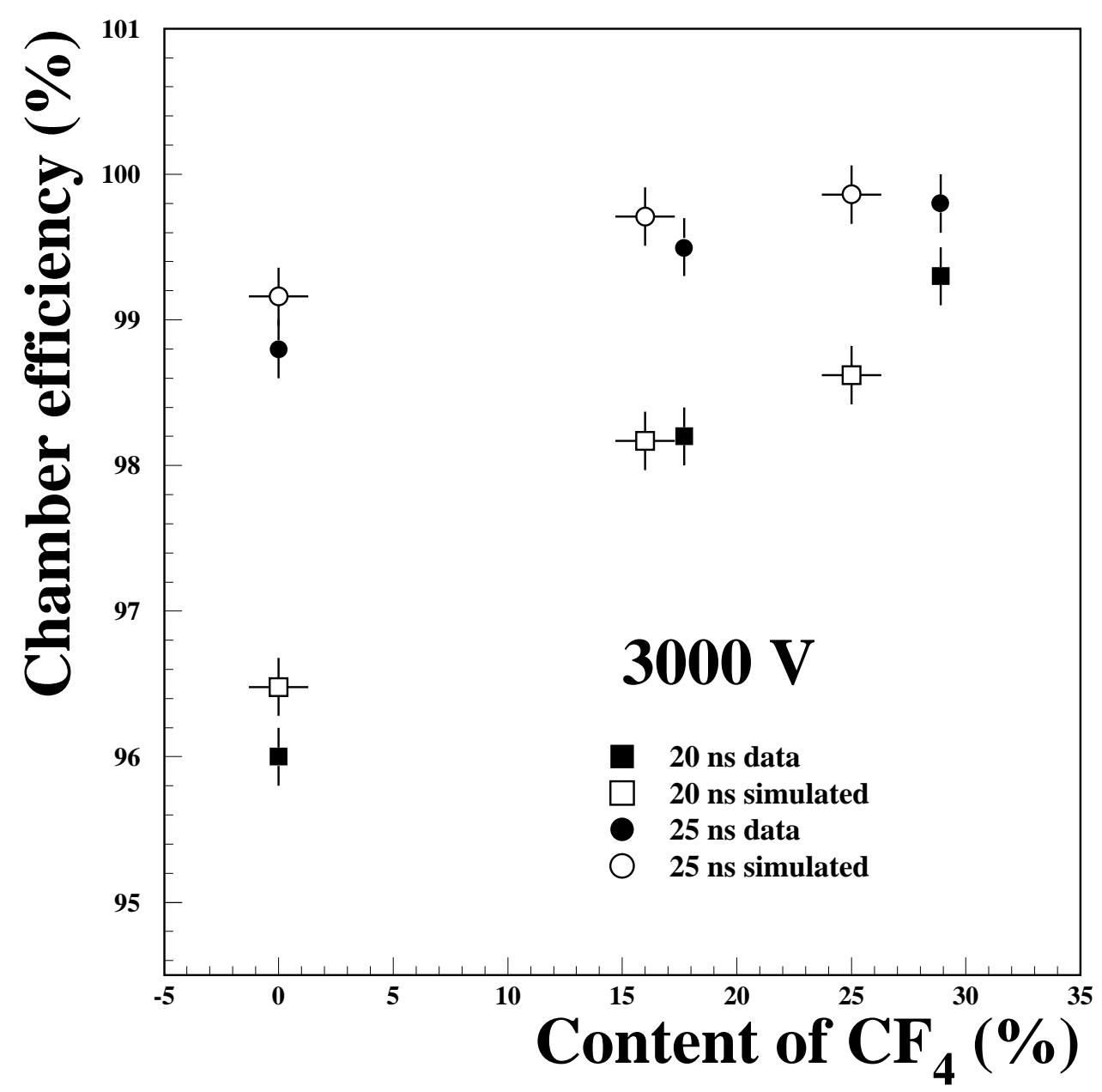

Fig. 5. Percentage of events 

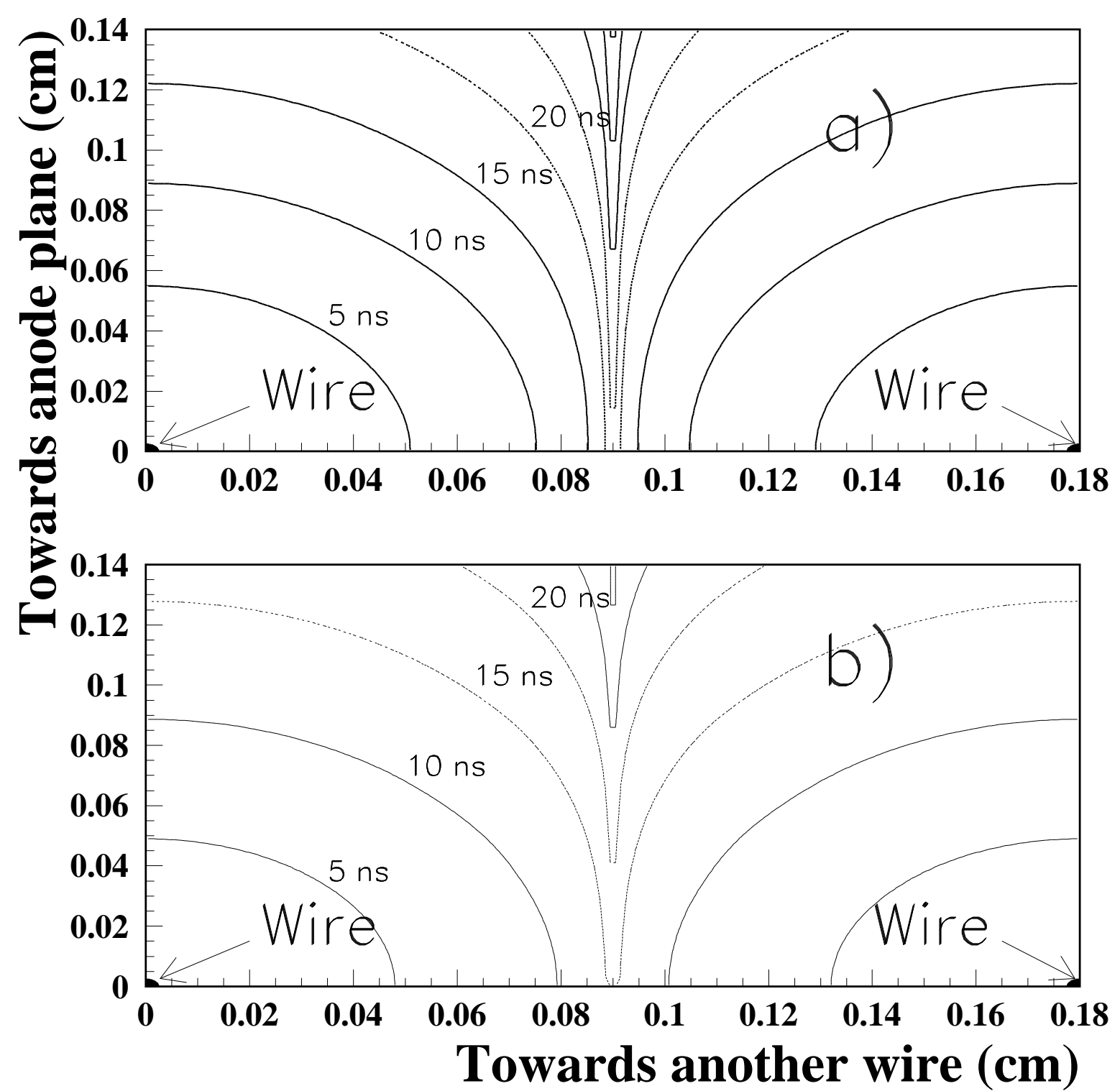

Fig. 6. Contours of equal arrival times for electrons in an elementary TGC cell, shown in $5 \mathrm{~ns}$ steps, as calculated by GARFIELD with $3000 \mathrm{~V}$ potential on the anode wire, for n-pentane- $\mathrm{CO}_{2}$ (45:55) (a) and for n-pentane- $\mathrm{CO}_{2}-\mathrm{CF}_{4}$ (45:30:25) (b).

The arrival time of the first cluster has some dependence on the angle of the track with respect to the wire plane. The path length of tracks traversing the chamber, and therefore the average number of clusters produced and the first cluster arrival time, depend somewhat on the track angle. However the dominant angular dependence of the arrival time arises from the proximity of the track path to either wire. The arrival time is the longest for those tracks passing equidistantly from two wires (see fig 6), the tracks passing at a large angle to the wire plane will, for some of their trajectory, necessarily approach 
close to one wire or another. Thus they are less likely to have produced all clusters in only the 'slow' region of the chamber.

In simulating the response to a radioactive source the angular distribution of tracks was assumed to be Gaussian with a width of five degrees, corresponding to the size of the source collimator. To reproduce test beam data with the simulation a constant, small incidence angle was used for all particles, as at the test beam itself.

The primary ionization clusters were produced along the track length. The number of clusters was generated according to a Poisson distribution with the mean calculated as described above. The position of any particular charge cluster along the particle track was randomly generated with a uniform distribution. For each primary charge, the arrival time corresponding to its position was found in the look-up tables. Loss of electrons by molecular attachment was neglected.

For each track, the earliest arrival time of all the primary clusters was taken to be the signal arrival time. With the assumption that the electron attachment is negligible, each cluster produces a signal and there was no need to deal with the number of electrons per given primary cluster.

The signal arrival time was convoluted with a Gaussian distribution that described the timing properties of the associated electronics. The width of the Gaussian was determined from the shape of the rising edge of the measured timing distributions and appeared to be constant for a given chamberamplifier combination. No correction for electron diffusion was found to be necessary, most probably due to small chamber size and strong electric fields.

This simplified approach enabled fast calculation of TGC timing properties for a given gas mixture and applied high voltage. A comparison with experimental data obtained with a collimated beam of high energy particles[8] allows for a check of the validity of the approach as well as an experimental determination of the number of primary ionization clusters. Figure 7 shows the simulation results compared to the data of [8]. The two are in good agreement for a density of $42 \pm 8$ clusters/cm.

The fraction of events in these distributions in time intervals shorter than 25 ns, represents the efficiency of interest for the operation at LHC. Figure 5 shows a good agreement of the simulated efficiency for different gas mixtures with the measurements of section 4 . 


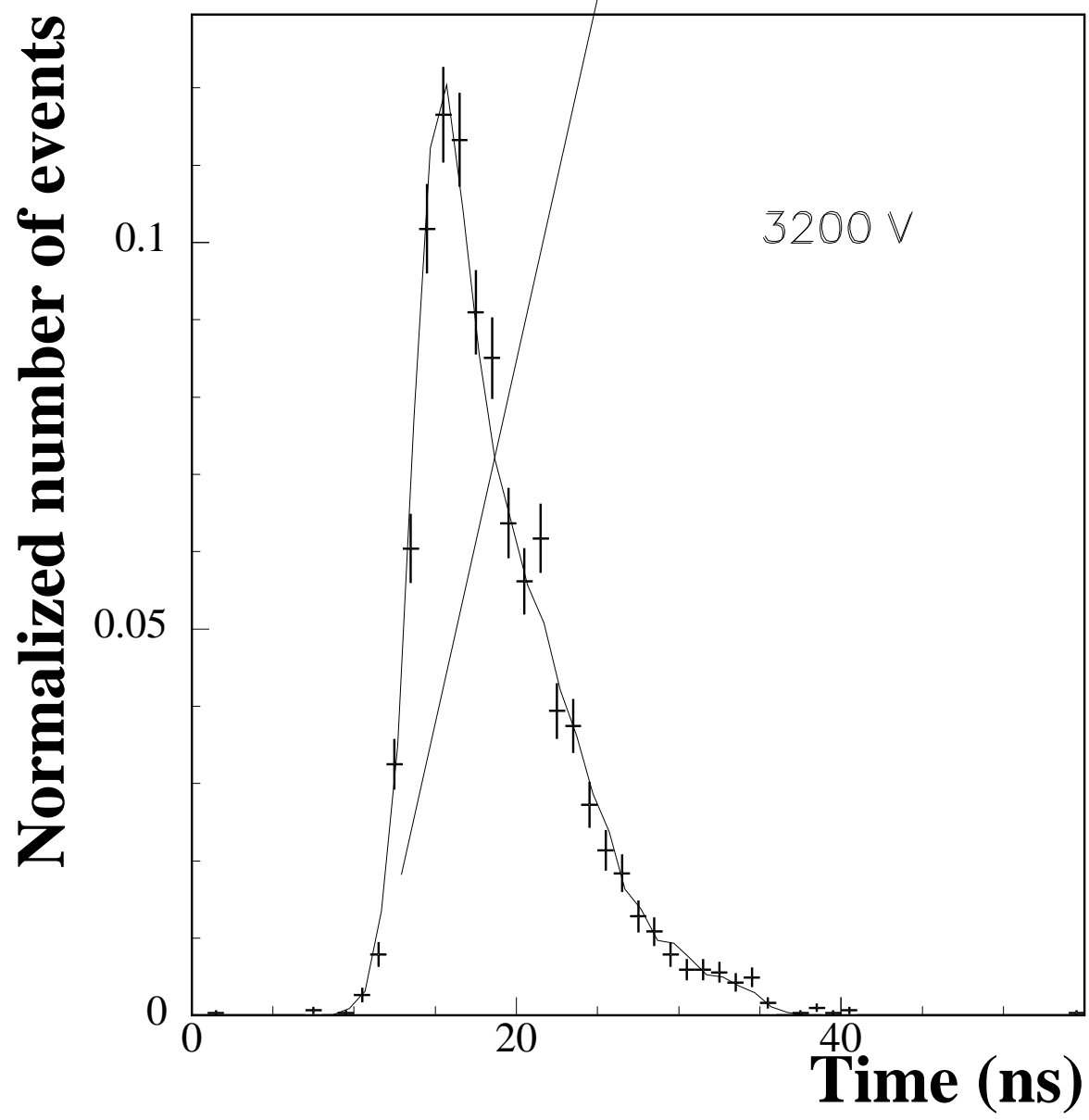

Fig. 7. Simulated arrival time of the first primary electrons in the thin gap c 
significant improvements of the speed of already existing detectors.

From the good agreement of the simulation and the measurements we conclude that the rather simple approach described above gives a reasonable description of the processes contributing to the chamber timing characteristics. This is a useful tool in chamber design and in the evaluation of potential gas mixtures.

\section{Acknowledgement}

The reported measurements were made possible by the funding from the Israel Ministry of Research and Israel Academy of Sciences, the Israel Council of Higher Education and the Lady Davis Fellowship Trust. The numerous discussions with R. Veenhof, the author of GARFIELD program package were very fruitful.

\section{References}

[1] ATLAS Technical Proposal, CERN-LHCC/94-43, LHC/P2 (15 Dec. 1994).

[2] CMS Technical Proposal, CERN-LHCC/94-38, LHC/P1 (15 Dec. 1994).

[3] G. Majewski et al. Nucl. Instr. and Meth.217 (1983) 265-271.

[4] G. Mikenberg et al. Nucl. Instr. and Meth.A265 (1988) 223-227.

[5] G. Bella et al. Nucl. Instr. and Meth.A252 (1986) 503-510.

[6] S. Dado et al. Nucl. Instr. and Meth.A252 (1986) 511-516.

[7] Y. Arai et al. Nucl. Instr. and Meth.A367 (1995) 398-401.

[8] ATLAS Collaboration, Muon Spectrometer Technical Design Report, CERN/LHCC 97-22, 1997 (in print).

[9] A. Breskin and R. Chechik, Nucl. Instr. and Meth.A252 (1986) 488-497.

[10] G. Malamud et al. Nucl. Instr. and Meth.A307 (1991) 83-96.

[11] W.W. Black, Nucl. Instr. and Meth.71 (1969) 317-327.

[12] GARFIELD, a drift-chamber simulation program, CERN Program Library W5050.

[13] F. Sauli,CERN Yellow Report77-04. 